\title{
Low-cost laser detection system with a 360-deg horizontal field of view
}

\author{
Marie Zandi $\odot$,* Kate Sugden, and David M. Benton $\odot$ \\ Aston University, Aston Institute of Photonic Technologies, Aston Triangle, Birmingham, \\ United Kingdom
}

\begin{abstract}
A low-cost laser detection system based on coherence detection has been developed and is able to detect weak, continuous laser sources even against bright background light. The system is composed of a Mach-Zehnder interferometer with one arm modified with a piezomounted mirror to modulate the path length. We introduce methods to determine the laser wavelength and to extend the horizontal field of view of the detector. To widen the field of view, a cone mirror is added to the system while the additional use of a camera allows the direction of the incoming laser beam to be studied. The wavelength from three different lasers is estimated with the use of the modulation amplitude of the piezo mirror. The preliminary results demonstrate that a 360-deg horizontal field of view can be achieved and that the direction of the laser beam can be determined with an estimated angular precision of \pm 5 deg. Moreover, the wavelength can be determined with a precision of $\pm 10 \mathrm{~nm}$. The system trades sensitivity for a larger field of view with the resultant detection sensitivity equal to $70 \mathrm{nW}$ (or $1 \mu \mathrm{W} \cdot \mathrm{cm}^{-2}$ ) at $635 \mathrm{~nm}$.

(C) The Authors. Published by SPIE under a Creative Commons Attribution 4.0 Unported License. Distribution or reproduction of this work in whole or in part requires full attribution of the original publication, including its DOI. [DOI: 10.1117/1.OE.60.2.027106]
\end{abstract}

Keywords: laser detection; coherence discrimination; cone mirror; laser direction; field of view; wavelength determination.

Paper 20201017 received Aug. 25, 2020; accepted for publication Jan. 27, 2021; published online Feb. 18, 2021.

\section{Introduction}

With the creation and the development of lasers in the early 1960s, the question of how to detect this technology has naturally followed. In the military, this question has raised particular interest since lasers have been used for decades for targeting, range finding, designation, and missile control. ${ }^{1}$ Laser warning receivers (LWRs) have been developed to identify the nature of the laser threats as well as the direction of irradiation. Most LWRs are effective at detecting high-energy laser pulses but they are less effective at detecting low-power lasers such as those used with laser beam riders or continuous wave $(\mathrm{CW})$ lasers. The last decade has seen the rise of a requirement to detect $\mathrm{CW}$ lasers in both military and nonmilitary scenarios. ${ }^{2}$ Our detection system is thus focused on the detection of $\mathrm{CW}$ lasers and requires a wide field of view, as it will allow a better coverage on the battlefield. Determining the wavelength would also be an advantage so as to identify the type of the threat.

Typically, LWRs are composed of three units: an optical unit, a detection unit, and a processing unit. ${ }^{3}$ The field of view of an LWR depends on the composition of the optical and/or detection unit but can be extended with additional optical components. For example, imaging laser detection systems (ILDS) are usually composed of a fish-eye lens and CCD or CMOS camera devices ${ }^{4}$ and can reach 180-deg of field of view. Oftentimes, several ILDS units need to be combined together to reach a 360-deg full azimuthal coverage. ${ }^{5}$ However, this is not a costeffective system. Interferometer-based detection systems are another type of LWR that make use of coherence detection, their field of view will be restricted by the size of the interferometer aperture and the optical path of the system. A static Michelson interferometer associated with an optical antenna has demonstrated a 45-deg horizontal coverage ${ }^{6}$ while a Fabry-Perot-based LWR has already achieved a horizontal field of view of $160 \mathrm{deg} .^{7}$ Sometimes, the field of view

*Address all correspondence to Marie Zandi, zandima@aston.ac.uk 
needs to be reduced to 120 deg in order to increase sensitivity in the system. ${ }^{8}$ Diffraction gratings can also be used as part of detection systems, and typically rely on the use of a CCD camera. In 2014, a fish-eye lens was combined with a sinusoidal amplitude grating and the system was able to achieve a 160-deg field of view. ${ }^{9}$

Among all those LWRs, interferometer-based systems and diffraction grating-based systems are the most reliable at determining the wavelength. However, they are mostly working with pulsed lasers, ${ }^{10,11}$ their field of view is limited, ${ }^{9}$ and/or they are complex and expensive. ${ }^{6,12}$

The laser detection system used for this work is composed of a modified Mach-Zehnder interferometer (MZI) and a balanced detector. ${ }^{2}$ It is able to detect weak, continuous laser sources against bright broadband backgrounds. In this paper, we expand on the application and practicality of this system. The determination of the input laser wavelength has been investigated. Furthermore, the field of view of the previous system has been measured and is equal to $\pm 3 \mathrm{deg}$, this value being due to the detector size (3-mm long) and the optical path of the system $(7.5 \mathrm{~cm})$. The aim of this work is to expand the field of view thereby making the device more practical. In addition to the field of view, the incoming beam direction has been studied as it is essential to determine the origin of the threats.

\section{Configuration of the Laser Detection System-Coherence Modulation}

\subsection{Principle of the Original System}

Unlike conventional laser detection systems based on the detection of enhanced brightness, the laser detection system presented here works by detecting light sources that have a significant coherence length. For this to work, the path difference between the two Mach-Zehnder arms is required to be longer than the coherence length of the background light, which is the case with a difference of few micrometers in length-determined by the spectral width detected by the system. A piezo-mounted mirror replaces one of the two reflective mirrors of the original MZI to modulate the path length in one arm. Thus, the path difference combined with the modulating element causes the output signal to be modulated at a known frequency only if the input light has a longer coherence length than the path difference. In other words, detecting a modulating signal will indicate the presence of laser light in the system.

The detection part is composed of two balanced photodiodes at the outputs of the interferometer and a data acquisition unit (USB-6341 Multifunction I/O Device) used to digitize and send the data to a computer. A schematic representation of the detection system is shown in Fig. 1. The plano-convex lens (focal length $f=7.5 \mathrm{~cm}$, aperture size $=2.5 \mathrm{~cm}$ ) helps to focus the laser beam on the two photodetectors. The beam splitters and the mirrors are 1-cm long.

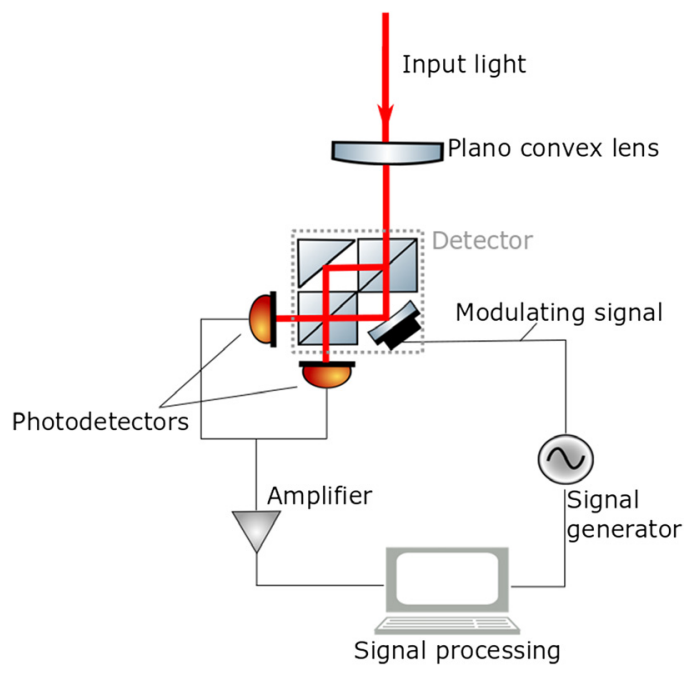

Fig. 1 Configuration of the Mach-Zehnder laser detection system. 


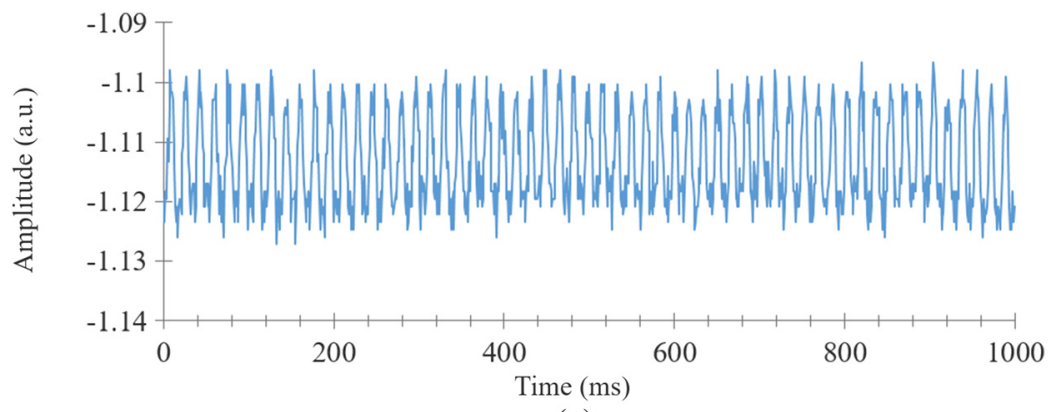

(a)

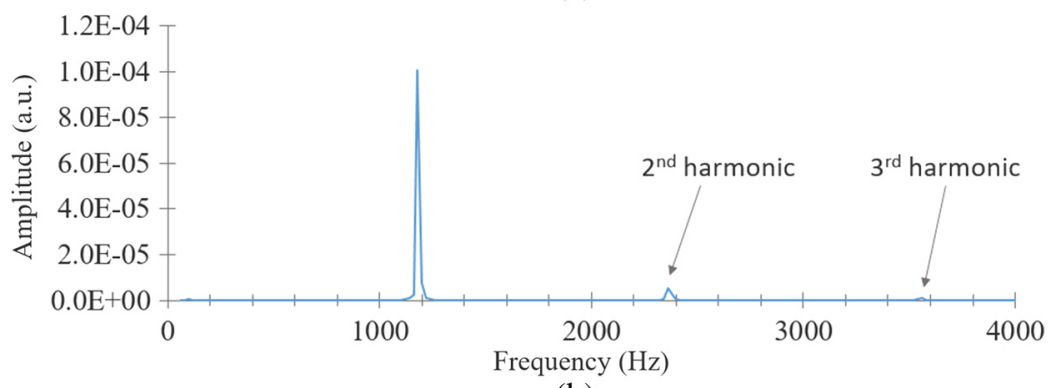

(b)

Fig. 2 (a) Example of an output CW signal modulated at $1200 \mathrm{~Hz}$ received by the balanced photodiodes. (b) Fourier transform of the signal.

The total optical path length from the focusing lens to the photodetectors is equal to $7.5 \mathrm{~cm}$ and this limits the field of view. The photodiodes are Si type and have a wavelength detection range from 350 to $1100 \mathrm{~nm}$. For this experiment, we typically used a 635-nm laser diode, the piezo mirror was modulated at a frequency of $1.2 \mathrm{kHz}$ and at an amplitude of $2.5 \mathrm{~V}$.

To detect modulating signals at the output, the voltage from a transimpedance amplifier was sampled and a Fourier transform analysis is made: peaks in the Fourier transform at the modulation frequency (and its harmonics) are evidence of coherent input. A rolling average spectrum was generated by summing consecutive frequency spectra. The graph in Fig. 2 gives an example of a signal modulated at $1200 \mathrm{~Hz}$ detected by the two balanced photodiodes and the Fourier transform applied to the signal where the second and third harmonics are visible.

\subsection{Laser Detection Theory}

This theory is a more complete and explanatory version of the theory that was presented in previous work. ${ }^{2}$ For an MZI with a path length modulated with a sinusoid of frequency $f_{m}$, the intensity modulation at the output port is given by

$$
I_{d}=\frac{1}{T}(E(1-R(\lambda)) R(\lambda) \gamma)^{2}[1+\cos (k(\Delta L(t)))]
$$

where $R(\lambda)$ is a wavelength-dependent reflectivity for the beamsplitters, $E$ is the light field amplitude, $\gamma$ is a factor representing reflection loss, $T$ is the sampling time, $k=2 \pi / \lambda$, and $\Delta L$ is the path length difference between the length of the two arms $L_{1}$ and $L_{2}$.

$$
\Delta L(t)=L_{1}-L_{2}(0)-v_{m} p \sqrt{2} \sin \left(2 \pi f_{m} t\right)-v_{\text {off }} p \sqrt{2},
$$

where $v_{m}$ is the voltage modulation amplitude, $v_{\text {off }}$ is a dc offset voltage applied to the mirror, and $p$ is the response of the piezo in $\mu \mathrm{m} / \mathrm{V}$. Combining constant terms into a factor $C$, the detector intensity can be written as

$$
I_{d}=\frac{1}{T}(E(1-R(\lambda)) R(\lambda) \gamma)^{2}\left[1+\cos \left(k\left(v_{m} p \sqrt{2} \sin \left(2 \pi f_{m} t\right)+C\right)\right)\right]
$$


The Term $C$ is in fact a slowly varying term which also accounts for drift of the interferometer and thus affects the phase of the cosine term $(k C)$ producing a sinusoid as it varies beyond $\pi / 2$. We simplify using $I_{0}(\lambda)=\frac{1}{T}(E(1-R(\lambda)) R(\lambda) \gamma)^{2}$ and $\beta=k v_{m} p$ :

$$
I_{d}=I_{0}(\lambda)\left[1+\cos \left(\beta \sin \left(2 \pi f_{m} t\right)+\beta C\right)\right] .
$$

Using the trigonometric identity $\cos (A+B)=\cos (A) \cos (B)-\sin (A) \sin (B)$, we express this as a combination of sine and cosine contributions

$$
I_{d}=I_{0}(\lambda)\left[1+a\left(\cos \left(\beta \sin \left(2 \pi f_{m} t\right)\right)\right)+\sqrt{\left(1-a^{2}\right)} \sin \left(\beta \sin \left(2 \pi f_{m} t\right)\right)\right]
$$

where $a=\cos (\beta C)$ is effectively a phase-dependent distribution factor. Using the Bessel function identities,

$$
\begin{aligned}
& \sin (z \sin \theta)=2 \Sigma_{k=1}^{n}(-1)^{k} J_{2 k+1}(z) \sin ((2 k+1) \theta), \\
& \cos (z \sin \theta)=J_{0}(z)+2 \Sigma_{k=1}^{n}(-1)^{k} J_{2 k}(z) \sin (2 k \theta),
\end{aligned}
$$

where the functions $J_{k}$ are Bessel functions of the first kind, we get

$$
\begin{aligned}
I_{d}= & I_{0}(\lambda)\left[1+a\left[J_{0}(\beta)-2 J_{2}(\beta) \sin (2 \pi 2 f t)+2 J_{4}(\beta) \sin (2 \pi 4 f t)+. .\right]\right. \\
& \left.+\sqrt{\left(1-a^{2}\right)}\left(-2 J_{1}(\beta) \sin (2 \pi f t)+2 J_{3}(\beta) \sin (2 \pi 3 f t)+. .\right]\right]
\end{aligned}
$$

the intensity at the detector can thus be represented in terms of harmonics of the modulation frequency where the odd and even harmonics vary as independent sets but with fixed internal ratios. The effect of slow offset drift can thus adjust the relative power in odd and even frequencies through the factor $a$. This can be actively controlled using a voltage offset on the piezo mirror. Setting $a=0$ by controlling $V_{\text {off }}$ produces the odd harmonics and removes the "lost" power that goes into the $J_{0}(\mathrm{DC})$ term.

\subsection{Implementation of a Wide Horizontal Field of View}

A wide horizontal field of view is achieved using a cone mirror (Edmund Optics). It is composed of a cylindrical base and a cone top; the top has a coating allowing reflection of a good percentage of the light toward the direction perpendicular to the input light, as shown in Fig. 3(a). The cone mirror used in the experiment has a diameter of $1 \mathrm{~cm}$ and a total length of $1.5 \mathrm{~cm}$ and reflects $90 \%$ of the light at $635 \mathrm{~nm}$. A beam reflected from the cone mirror is widely distributed

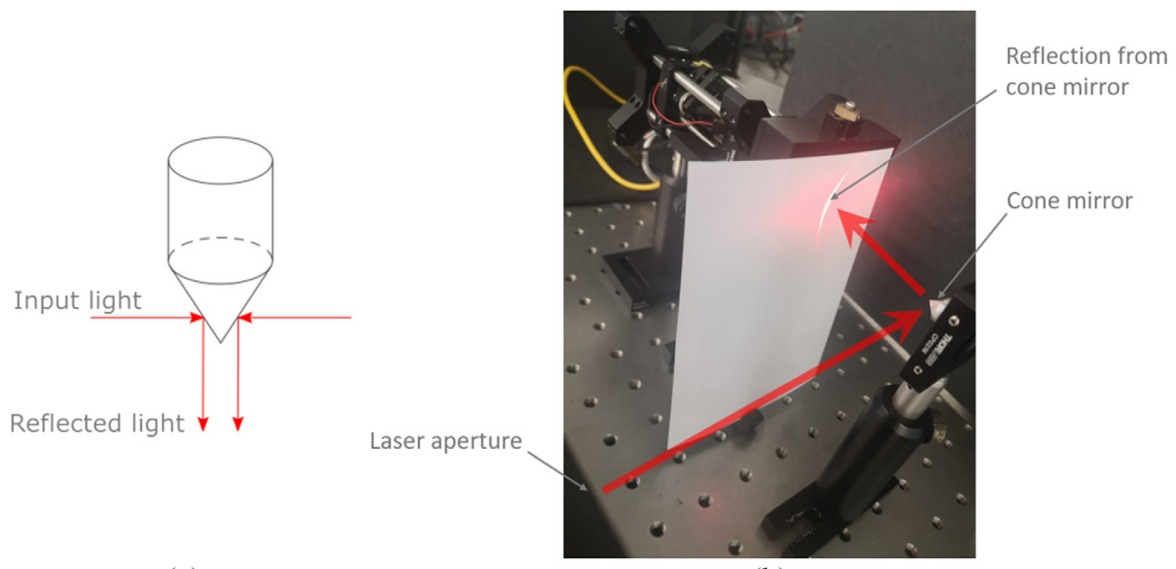

(a)

(b)

Fig. 3 (a) Reflection principle of the cone mirror. (b) Example of a beam reflected by the cone mirror. 


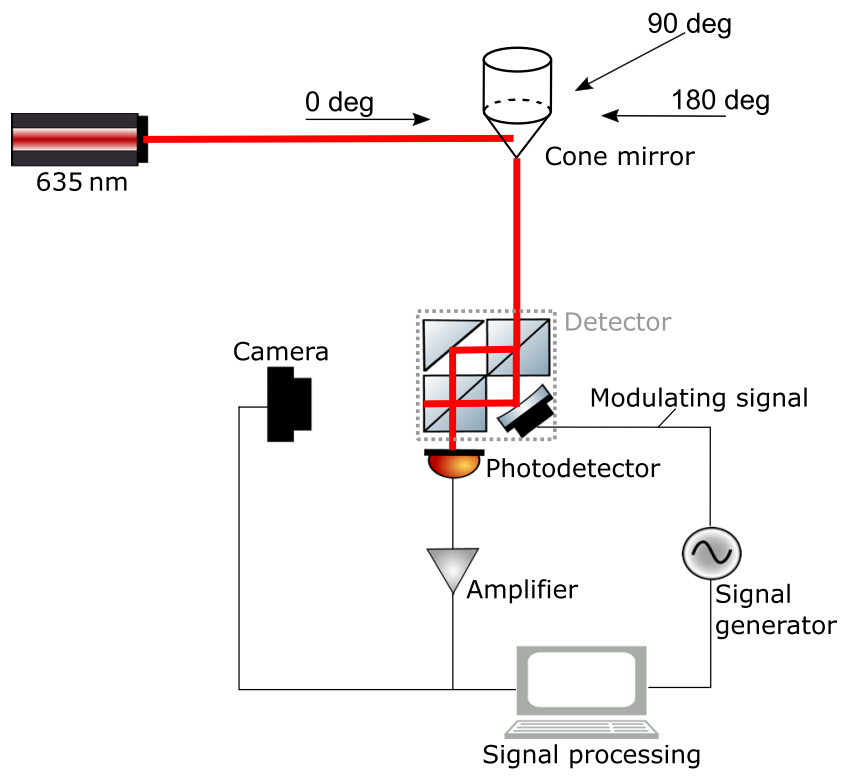

Fig. 4 Configuration of the laser detection system with the cone mirror.

in an arc, as can be seen in Fig. 3(b). The arc length depends slightly on the incident angle, and the incident angle can only vary by few degrees to get the best reflection from the cone mirror.

In this work, the previous detection system composed of two photodiodes has been modified: one of the outputs is used to measure the intensity of the modulating signal via one photodiode while the second output is used to determine the direction of the beam via a camera. Both functions can be performed at the same time. This will have an effect on the sensitivity, which is reduced without the benefit of a balanced detector system.

The cone mirror is positioned to direct light into the interferometer and the system with the cone mirror is aligned vertically as shown in Fig. 4. The camera is looking through the interferometer from one of the output ports and is focused on the cone mirror. To show the effectiveness of this approach, a laser was directed into the system at various angles spaced by $45 \mathrm{deg}$. The 0-deg angle was set as illustrated in the figure. The amplitude of the first harmonic and a camera image were recorded for each angle ( $0 \mathrm{deg}, 45 \mathrm{deg}$, $90 \mathrm{deg}, 135 \mathrm{deg}, 180 \mathrm{deg}, 225 \mathrm{deg}$, $270 \mathrm{deg}$, and $315 \mathrm{deg}$ ).

\section{Experimental Results}

\subsection{Determination of the Direction of Irradiation}

Captured images of the cone mirror when the laser is shone at 0 deg and 225 deg are presented in Fig. 5. As can be observed on the captures, the beam is visible on the surface of the cone mirror and the direction of the laser input can be inferred from its relative angular position. It should be noted that the laser spots on the cone mirror are not similar to each other for different angles, which is due to the experimental method: the laser was positioned manually around the cone mirror, which creates a possibility of misalignment on the laser-cone mirror vertical plane. This would then cause a different incident angle on the cone mirror and thus create a difference in the light reflected by the cone mirror. There is possibly also a misalignment of the cone mirror axis with the interferometer axis.

While the first output of the interferometer is capturing the cone mirror images, the second output is simultaneously measuring the amplitude of the first harmonic of the signal. The results can be seen in Fig. 6, where both the amplitude of the first harmonic and the capture of the cone mirror are combined for each angle. In the graph, the radial distance represents the amplitude of the first harmonic. These results show that the first harmonic is measured for every laser input angle. Thus, it validates that the laser is detected as a modulating input at every given angle. 


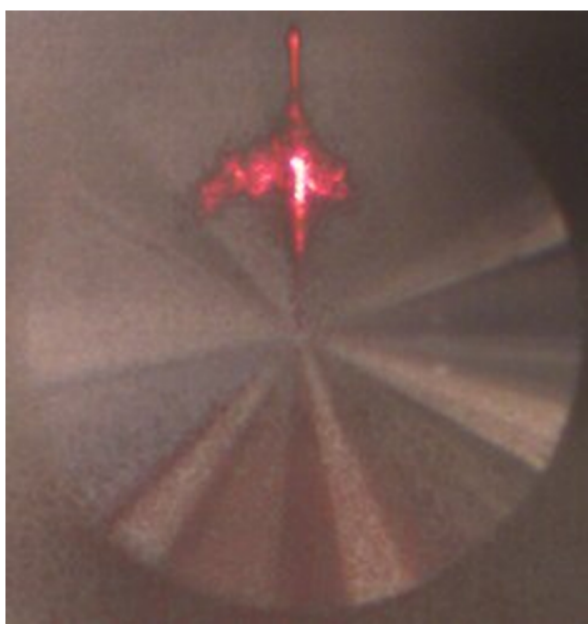

(a)

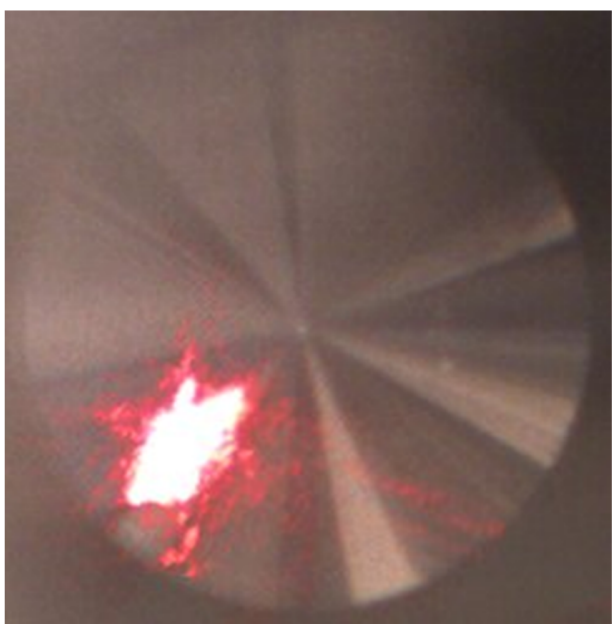

(b)

Fig. 5 Captured images of the cone mirror when the laser is positioned at 0 deg and 225 deg.

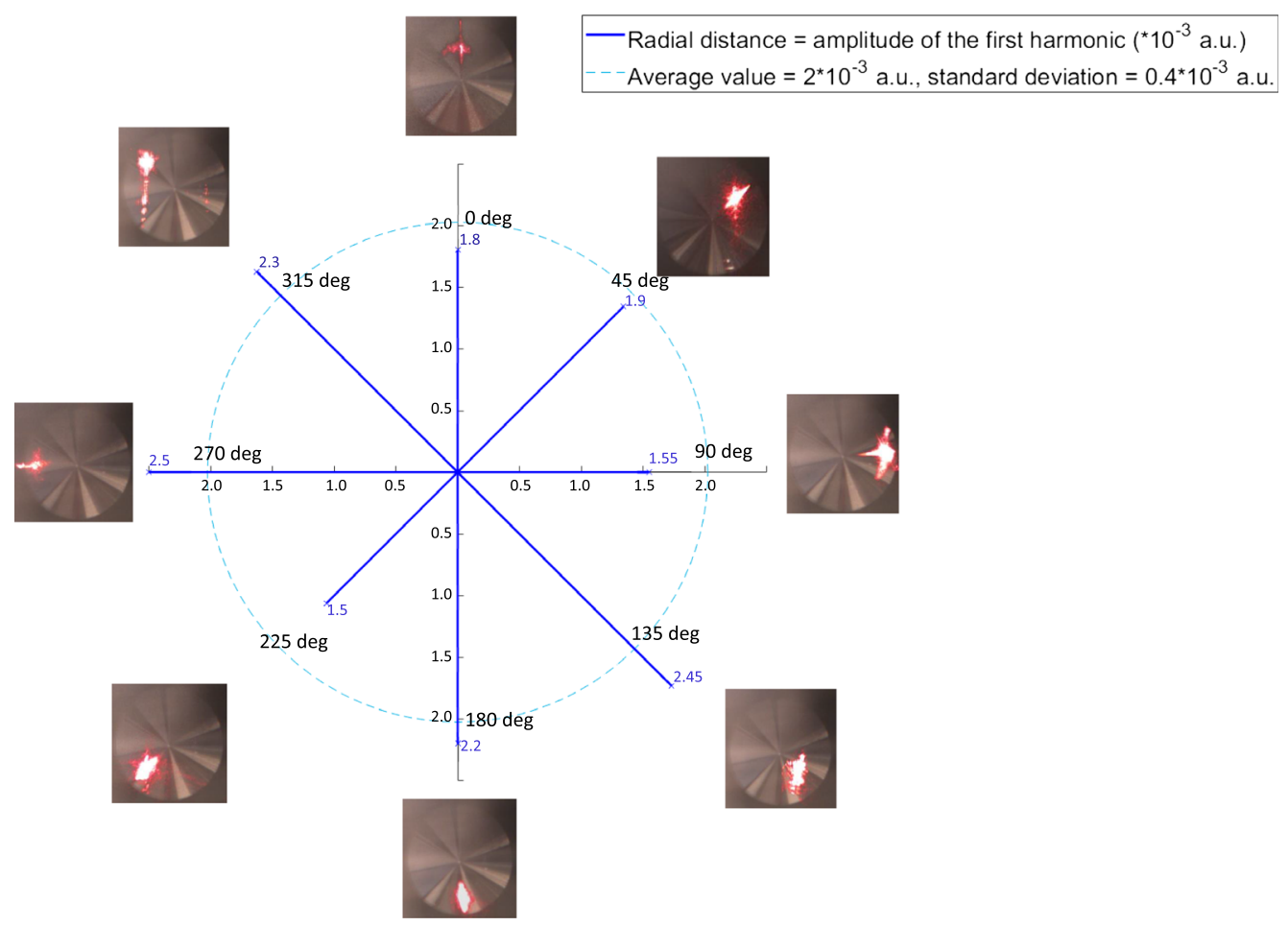

Fig. 6 Combined data of the first harmonic amplitude and the cone mirror image for every angle spaced by 45 deg.

Moreover, the measured amplitudes of the fundamental frequency proved to be high, implying that weak signals could still be detected. Sensitivity measurements with and without the cone mirror have been studied and are shown and discussed in Sec. 3.3. Finally, the captures shown in Fig. 6 also demonstrate that the direction of the laser beam can be determined from the camera at every angle. However, the capture of the cone mirror at 315 deg shows a deformed beam reflection, which would impact the accuracy in the determination of the irradiation direction. Nevertheless, if the center of mass of the beam is calculated for each capture, a better accuracy of the direction can be worked out. Thus, an estimated angular accuracy of \pm 5 deg in the direction of the laser can be asserted. 


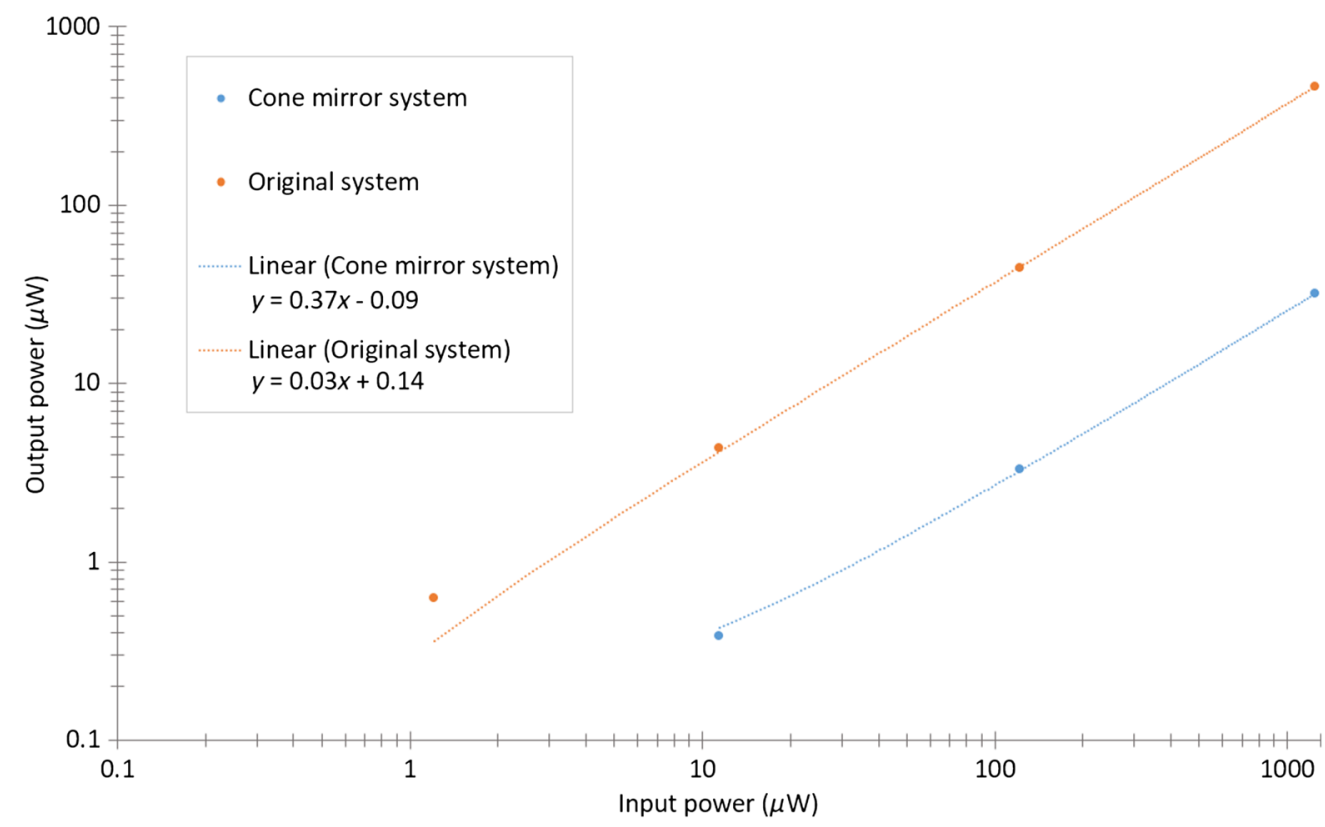

Fig. 7 Transmission efficiency comparison between the cone mirror and the original systems.

\subsection{Transmission Efficiency Measurements for Original and Cone Mirror Systems}

In this section, the difference in signal attenuation between the original system (see Fig. 1) and the cone mirror system (see Fig. 4) is analyzed. The signal attenuation is calculated by looking at the power of the laser beam before it enters the detector and after it leaves through the last beam splitter. To have a relevant comparison with the cone mirror system, the detection part of which is composed of one single photodiode, only one of the two outputs in the original system is measuring the power. The intensity of the laser diode is attenuated with neutral-density filters and a power meter is used to measure the input/output power level.

In Fig. 7, the input/output power ratio for the original system and for the cone mirror system are shown. This gives a transmission efficiency of $37 \%$ for the original system and of $3 \%$ for the cone mirror system. The original system had a maximum 50\% transmission efficiency to be expected as only one of the two output ports is used in the measurement, the additional efficiency loss is due to the optics in the MZI. In the cone mirror system, the additional losses are the result of the typical beam shape reflected from the cone mirror (see Fig. 3) since only a part of the beam can reach the detector input.

\subsection{Sensitivity Measurements for Original and Cone Mirror Systems}

The sensitivity corresponds to the minimum laser power required to detect a peak in the Fourier transform. Sensitivity measurements are done by measuring the signal-to-noise ratio $(\mathrm{S} / \mathrm{N})$ for different laser powers, the $\mathrm{S} / \mathrm{N}$ ratio is calculated by measuring the amplitude of the fundamental harmonic and by comparing it to the noise level in the Fourier transform. The results are plotted in Fig. 8.

These results showed that the cone mirror system is able to detect a signal down to around $70 \mathrm{nW}$ (or $1 \mu \mathrm{W} \cdot \mathrm{cm}^{-2}$ ); this is the ultimate sensitivity, the detected power or irradiance for which $\mathrm{S} / \mathrm{N}=1$. These are quite interesting results; they attest that the system when combined with a cone mirror is still able to detect weak signals under $100 \mathrm{nW}$. Moreover, it can be seen that without a cone mirror (original system), the ultimate sensitivity is around $2 \mathrm{nW}$ or $0.03 \mu \mathrm{W} \cdot \mathrm{cm}^{-2}$. Thus, we can see that we have traded the benefit of a wide detection field of view for a reduced sensitivity. Nevertheless, the system retains a useful sensitivity level. 


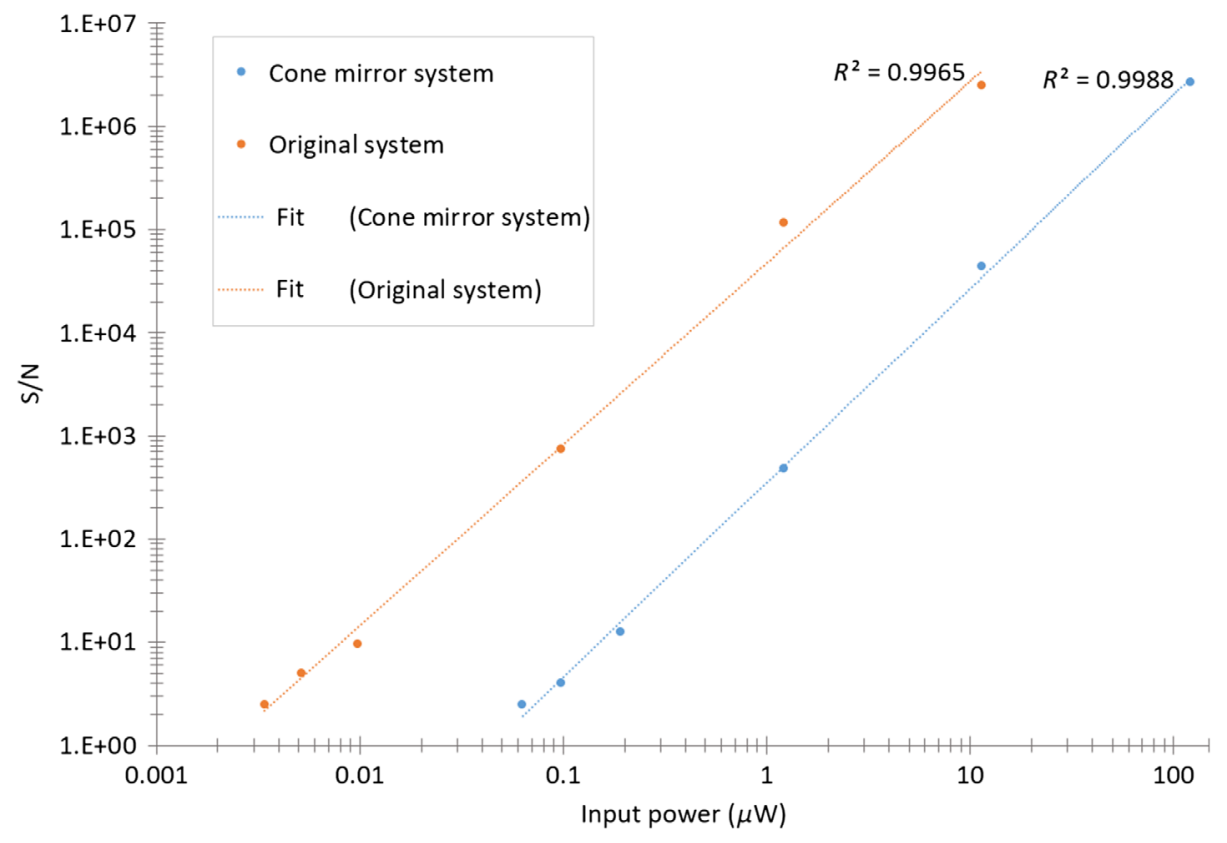

Fig. 8 Sensitivity comparison between the cone mirror and the original systems.

\subsection{Confirmation of Laser Detection System Principle}

To demonstrate that the modified MZI combined with the cone mirror detects only coherent sources, a light-emitting diode (LED) and a laser were both directed toward the cone mirror. Both were at similar wavelength $(635 \mathrm{~nm})$ and can be seen on the camera image of the cone mirror in Fig. 9. The LED appears significantly brighter on the camera image; however, no change in the amplitude of the first harmonic is seen when the LED is turned on and off. As expected, the LED contributes nothing to the modulation signal.

\subsection{Wavelength Determination}

In our prior work, ${ }^{2}$ the potential of determining the wavelength of the laser light was proposed. This could be done by measuring the ratio of the first and third harmonics of the modulation frequency within the detector signals. Like many concepts, they do not always work in the real world. Measurements of laser wavelength were attempted using harmonic ratios, but the results were found to be unreliable. The first plot in Fig. 10 shows the estimated wavelength evolving with time as a blue laser at $405 \mathrm{~nm}$ is detected by the system. The plot in the lower panel shows the power at the modulation frequency and its second harmonic. As the interferometer drifts, power is divided between the odd and even harmonics and the powers develop in antiphase-as was outlined in the theory section. The sharp discontinuities represent manual adjustments to the offset voltage applied to the piezo to minimize the second harmonic power. The timescale represents around $10 \mathrm{~min}$ of observation. Shorter wavelengths actually represent the best conditions

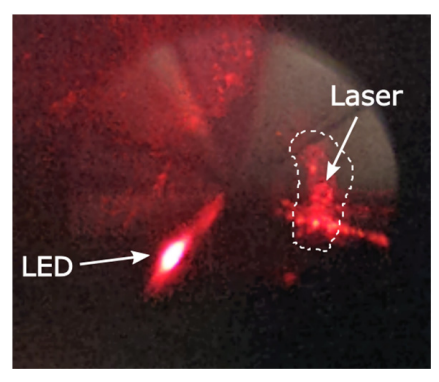

Fig. 9 Captures of the LED and the laser beam reflections on the cone mirror. 


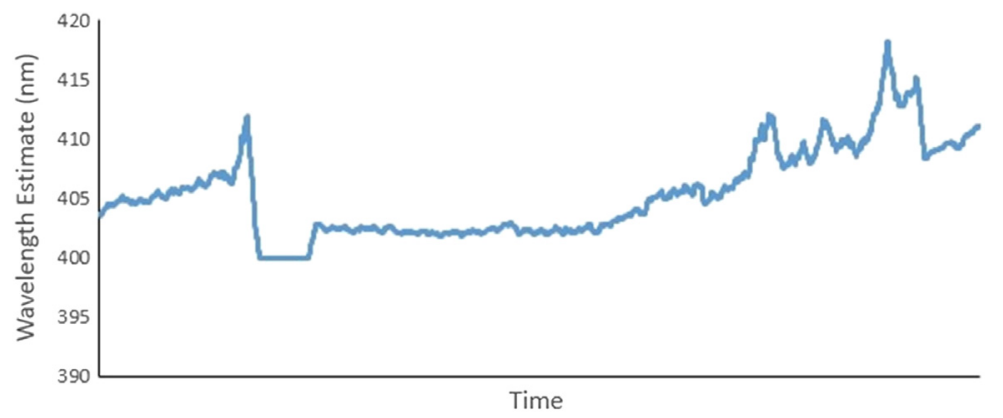

(a)

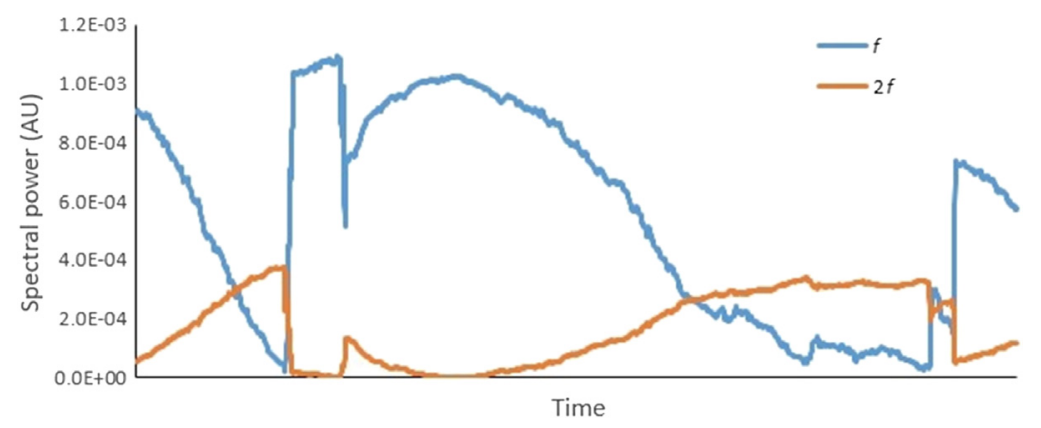

(b)

Fig. 10 (a) Wavelength estimated using harmonic ratios varying with time for a blue laser at $405 \mathrm{~nm}$. (b) The power in the fundamental modulation frequency and its second harmonic.

for wavelength estimation as they have larger third harmonic powers. The problem lies in the precision with which digitization can be performed. The DAC being used was a 14-bit precision device measuring $\pm 10 \mathrm{~V}$, giving a measurement resolution of $1.2 \mathrm{mV}$. Assuming a third harmonic power present at this resolution, we can estimate the power needed in the fundamental frequency to produce this. We consider the use of a red wavelength at $635 \mathrm{~nm}$ as piezo modulation amplitudes are set for longer wavelengths. The ratio of harmonic amplitudes, $3 f: f$, at a wavelength of $635 \mathrm{~nm}$ is $R=0.022$, which requires an amplitude of $56 \mathrm{mV}$ for the fundamental frequency. We can calculate the expected $3 f: f$ ratios at neighboring wavelengths $( \pm 10 \mathrm{~nm})$ and use this to determine the required size of the $3 f$ signal needed to determine the wavelength to this accuracy. This suggests that the $3 f$ signal must be 8 quantization units, thus requiring a fundamental signal of $8 \times 56 \mathrm{mV}=440 \mathrm{mV}$. This is the minimum power needed to determine this wavelength with an accuracy of $\pm 10 \mathrm{~nm}$. This does not take account of noise on the signal, which was of order $3 \mathrm{mV}$, arising from intensity noise in the laser and contributed to inaccuracy in the wavelength determination. Clearly even though this interferometric technique is capable of sensitivity in the $\mathrm{nW}$ regime, wavelength determination via harmonic ratio requires relatively strong sources.

An alternative method of determining wavelength has been investigated. This makes use of the response being related to the amplitude of the mirror modulation. The amplitude of the fundamental frequency is represented by the term $J_{1}(\beta)$ which varies with modulation amplitude. By scanning the amplitude of the voltage applied to the mirror modulation, we can locate the peak response of the system which will be wavelength dependent. The maximum voltage that can be applied is limited to $3.5 \mathrm{~V}$ due to the power supply voltages of the driving amplifiers for the piezo mirror modulation signal. The plot in Fig. 11 shows the measured response at the modulation frequency for three wavelengths as the modulation amplitude voltage applied to the piezo is varied. This clearly shows the wavelengths peaking at different amplitudes. Voltage steps of $0.1 \mathrm{~V}$ were used and modeling suggests that by just assessing the peak voltage value a wavelength precision of around $\pm 10 \mathrm{~nm}$ would be achieved. Fitting a function to measured values would give a much better estimate. Bessel functions were fit to the data but required some wavelength-dependent tweaking and have not been applied to an unknown wavelength. This approach is clearly slower than the harmonic ratio approach as it requires a capture for 


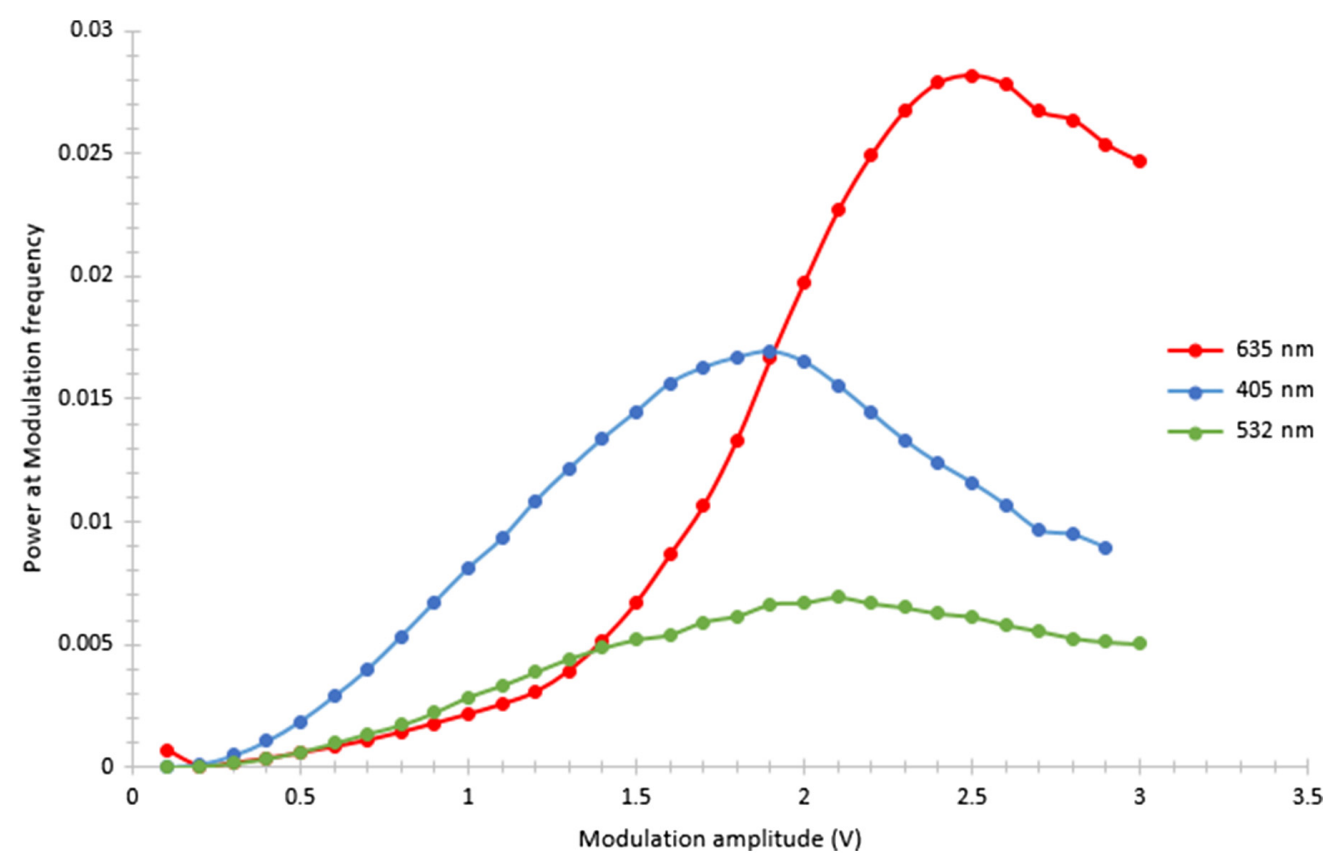

Fig. 11 The power of signal at the modulation frequency for three wavelengths as the amplitude of the modulation voltage is varied.

each amplitude point. This may not be practical although with a well-understood system fewer points would be needed to determine the amplitude variation. However, this approach is not limited to strong signals and can be used with significantly weaker sources as the predominant modulation power is measured.

\section{Conclusion}

It is relatively uncommon to find LWRs that can detect CW lasers, determine the laser wavelengths, and have a wide field of view. In this paper, we presented a means to widen the field of view of an MZI detection system, to determine the laser direction and the laser wavelength of a CW laser. A cone mirror was introduced into the system and allowed a CW laser to be discriminated from background light for a range of input angles, demonstrating a 360-deg field of azimuthal detection. With the use of a camera at one of the two system outputs, images of the cone mirror are captured. They show the position of the laser source on the cone mirror from which the source direction can be inferred. The direction determination method does not impact the sensitivity of the system excessively since using only one output of the MZI with a photodiode is enough to detect a laser signal down to $70 \mathrm{nW}$ (or $1 \mu \mathrm{W} \cdot \mathrm{cm}^{-2}$ ). Moreover, if an LED is reflected together with a laser from the cone mirror, it appears that the LED is not detected by the system, confirming the ability to discriminate lasers from non-laser sources. Finally, the determination of the wavelength is possible with a precision of $\pm 10 \mathrm{~nm}$ with a basic incremental amplitude scan and could be much improved by fitting a function to a well-characterized system.

Overall, this modified detection system comes with a high sensitivity and a wide spectral range (in this case, the photodiode has a spectral range from 350 to $1100 \mathrm{~nm}$ ). The spectral range could be extended to infrared with suitable optics and IR detectors. The acquisition time of the detector is less than $1 \mathrm{~s}$ for weak sources which are integrated, less than $100 \mathrm{~ms}$ for stronger sources. The use of a camera for direction finding could produce issues when multiple sources are present, as this responds to brightness. In this work, an LED and laser at the same wavelength were indistinguishable to the camera and sufficed to say that one of the sources was a laser without being specific. In general, if the sources were of different wavelengths, this may be sufficient to discriminate them (if a color camera is used). Moreover, the imaging of the beam in the cone mirror requires some image analysis to increase the angular accuracy in the direction determination, so far being at $\pm 5 \mathrm{deg}$. 


\section{Acknowledgments}

The authors would like to thank the Defence Science and Technology Laboratory for the funding as part of the Future Sensing and Situational Awareness program. This paper has some results previously reported in an SPIE Proceedings paper in $2019 .{ }^{13}$

\section{References}

1. J. Dubois and F. Reid, "Detecting laser sources on the battlefield," Proc. SPIE 6796, 67962F (2007).

2. D. Benton, "Low-cost detection of lasers," Opt. Eng. 56(11), 114104 (2017).

3. S. M. Nejad, H. Arab, and N. R. Sheshkelani, "Analysis of new laser warning technologies to propose a new optical subsystem," Iran. J. Electr. Electron. Eng. 14(3), 213-221 (2018).

4. B. Chen and W. Zhang, "Study on warning radius of diffuse reflection laser warning based on fish-eye lens," Proc. SPIE 8907, 89072Z (2013).

5. M. Dabrowski et al., "Laser warning receiver LWR-H," Proc. SPIE 6598, 65980S (2007).

6. X. Li et al., "A new design for laser warning system," in Proc. 7th WSEAS Int. Conf. Signal, Speech and Image Process., pp. 187-189 (2007).

7. E. Siebert, "Analyzer for coherent radiation," Patent 4,309,108 (1982).

8. J. Russell, “Coherent laser warning system," Patent 6,151,114 (2000).

9. L. Wang et al., "Optimum design of wide angle laser detecting system based on fish eye lens and sinusoidal amplitude grating," Opt. Commun. 310, 173-178 (2014).

10. L. Fu and Z. G. Hong, "Design of laser warning receiver based on DSP," in Asia Pac. Conf. Postgraduate Res. Microelectron. Electron., pp. 325-328 (2009).

11. A. D. McAulay, "Detecting modulated lasers in the battlefield and determining their direction," Proc SPIE 7336, 73361J (2009).

12. M. B. Sinclair and W. C. Sweatt, "Laser warning receiver to identify the wavelength and angle of arrival of incident laser light," Patent 7,683,310 B1 (2010).

13. M. A. Zandi, D. M. Benton, and K. Sugden, "Developments in low-cost laser detection: wide field of view implementation and direction determination," Proc. SPIE 11161, 111610H (2019).

Marie Zandi received her engineering degree in photonics from the Advanced National College of Applied Science and Technology (ENSSAT), France, in 2017. She is a PhD candidate at Aston University, Birmingham, UK. Her current research interests include laser detection techniques and optical aberration corrections. She is a member of SPIE.

Kate Sugden received her BSc degree in physics from Birmingham University, UK, in 1989 and her MSc degree in optoelectronics and laser devices from St Andrews University, UK, in 1991, and her PhD in photonics from Aston University, UK, in 1996. She returned to the Aston Institute of Photonic Technologies in 2004. Since 2018, she has been the deputy dean for Enterprise and International in the School of Engineering and Applied Science at Aston University. Her current research interests include optical fiber sensing, laser material processing, OCT metrology, laser detections, and 3D printing. She is a member of the Institution of Engineering and Technology (IET) and Institute of Physics (IoP).

David M. Benton graduated in physics from the University of Birmingham in 1989. He completed a PhD in laser spectroscopy for nuclear physics in 1994 and then conducted postdoctoral research in positron emission tomography and then laser spectroscopy for nuclear physics, all at the University of Birmingham. In 1998, he joined DERA which became QinetiQ, where he worked on a variety of optical projects. He was the leader of a group building quantum cryptography systems and was involved in a notable $140 \mathrm{~km}$ demonstration in the Canary Islands. He became chief scientist for L-3 TRL in 2010, working on photonic processing techniques for RF applications. He is now at Aston University with a variety of interests, including novel encoding techniques, gas sensing, and laser detection techniques. He is a member of SPIE. 